Document downloaded from:

http://hdl.handle.net/10251/141974

This paper must be cited as:

Olmo-Juan, N.; Demazière, C.; Barrachina, T.; Miró Herrero, R.; Verdú Martín, GJ. (08-2).

PARCS vs CORE SIM neutron noise simulations. Progress in Nuclear Energy. 115:169-180. https://doi.org/10.1016/j.pnucene.2019.03.041

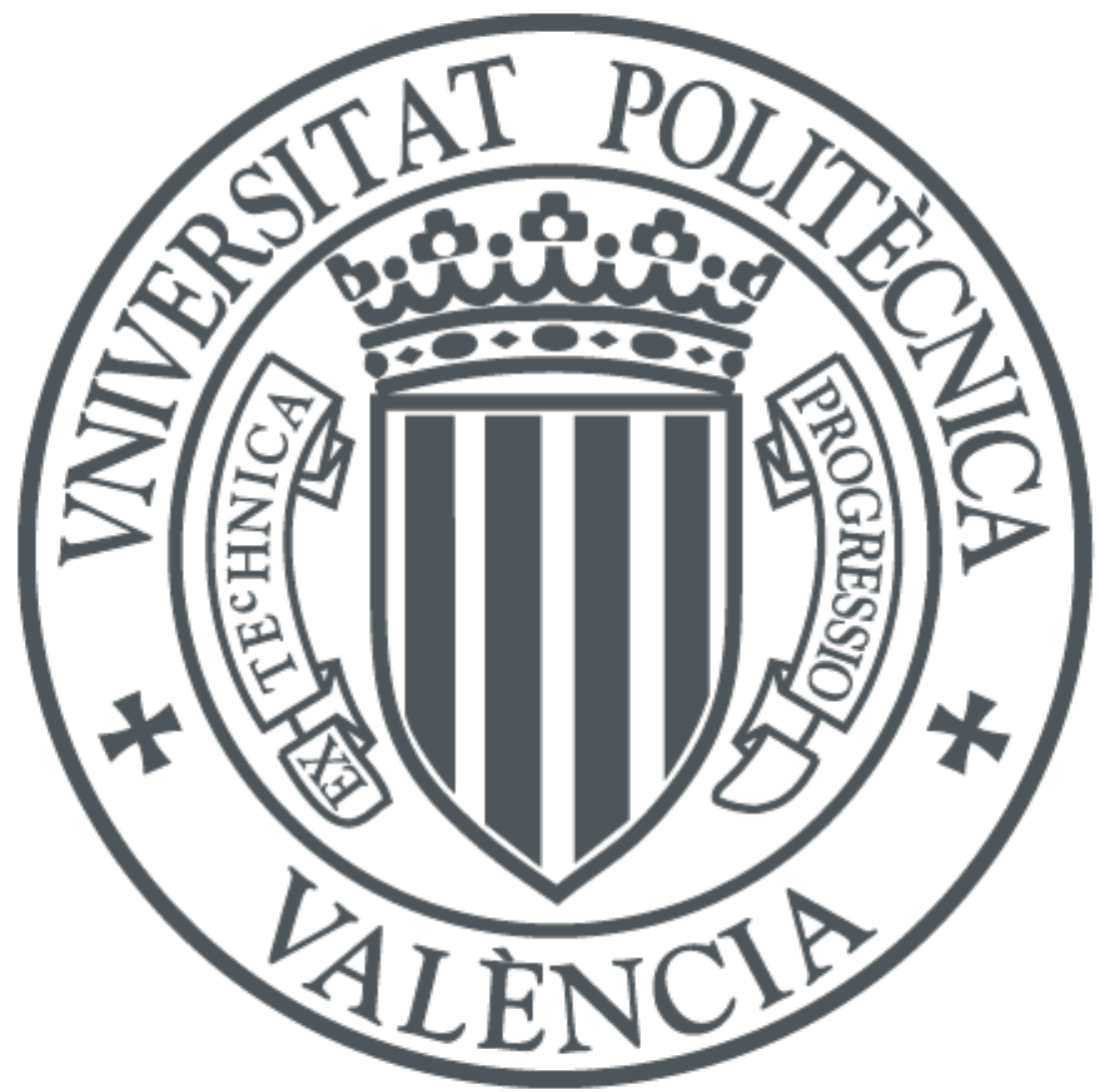

The final publication is available at

https://doi.org/10.1016/j.pnucene.2019.03.041

Copyright Elsevier

Additional Information 


\title{
PARCS vs CORE SIM NEUTRON NOISE SIMULATIONS.
}

\author{
N. Olmo-Juan ${ }^{1}$, C. Demazière ${ }^{2}$, T. Barrachina ${ }^{1}$, R. Miró ${ }^{1}$ and G. Verdú ${ }^{1}$ \\ ${ }^{1}$ Institute for Industrial, Radiophysical and Environmental Safety (ISIRYM) \\ Universitat Politècnica de València. Camí de Vera s/n 46021, València, Spain
}

${ }^{2}$ Chalmers University of Technology, Department of Applied Physics, Division of Subatomic and Plasma Physics, SE-412 96 Gothenburg, Sweden

\author{
nioljua@iqn.upv.es,demaz@chalmers.se,tbarrachina@iqn.upv.es, rmiro@iqn.upv.es, \\ gverdu@iqn.upv.es
}

\begin{abstract}
In a nuclear reactor, even operating at full power and steady-state conditions, fluctuations are detected in the recording of any process parameter. These fluctuations (also called noise) could be of various origins, such as, turbulence, mechanical vibrations, coolant boiling, etc. The monitoring and complete comprehension of those parameters should thus allow detecting, using existing instrumentation and without introducing any external perturbation to the system, possible anomalies before they have any inadvertent effect on plant safety and availability.

In order to reproduce and study the induced neutron noise in a nuclear reactor core, it is compulsory to develop suitable tools. Existing time-domain codes were originally not developed for this type of calculations. Modifications of those codes and the development of an associated intricate methodology are necessary for enabling noise calculations. This involves, in some cases, changes in the source code and the development of new auxiliary tools to ensure accurate reproductions of the core behavior under the existence of a neutron noise source.

In the proposed work, the time-domain neutron diffusion code PARCS is used to model the effect of stationary perturbations representative of given neutron noise sources. In order to validate the feasibility of the time-dependent methodology thus developed, comparisons with the results of simulations performed in the frequency domain, using the CORE SIM tool, developed at Chalmers University of Technology, are performed.

The development of a few test cases based on a real reactor model are undertaken as the basis for such comparisons and a methodology aimed at assessing the time-domain simulations versus the frequency-domain simulations is established. It is demonstrated that PARCS, although not primarily developed for neutron noise calculations, can reproduce neutron noise patterns for reasonable frequencies. However, it is also observed that unphysical results are occasionally obtained.
\end{abstract}

KEYWORDS: time domain, frequency domain, diffusion equation, noise analysis

\section{INTRODUCTION}

The induced neutron noise in nuclear reactors can be related to many different phenomena that may have operational impacts and, in some cases, a safety relevance. In Pressurized Water Reactors (PWR) for example, the neutron noise can be induced, among other things, by vibrations of fuel assemblies, of control rods, and of the core barrel. Excessive vibrations, in addition to worsen core performance, might also lead to material degradation and impact plant safety. The monitoring of the effect of such 
fluctuations onto the neutron flux offers the possibility to early detect anomalies and if adequate inversion techniques are implemented, to make a fingerprinting of the anomaly [1].

To take full advantage of core monitoring based on the analysis of the neutron noise, a new Horizon2020 European project was launched in 2017. The project, called CORTEX (COre monitoring Techniques and EXperimental validation and demonstration) [2], gathers 20 different participants, from universities, research institutes to private companies. The main goal of the project is to develop neutron noise-based core monitoring techniques to be used in commercial reactors with the aim to help utilities to early detect and characterize anomalies.

For the analysis of the neutron noise in nuclear reactors, two different approaches can be applied: analysis in the time domain or in the frequency domain. In either case, the analysis requires the prior determination of the reactor transfer function, which gives the reactor response to predetermined perturbations. Once this reactor transfer function is available, it is thereafter possible, at least formally, to retrieve from the induced neutron noise the driving perturbation.

The modelling in the frequency-domain of the reactor transfer function is usually done using dedicated tools, solving separately the static neutron flux and the neutron noise. In the time-domain, simulations could be carried out using existing neutron kinetics code (without thermal-hydraulic feedback), which by default do not separate the static and the fluctuating parts. Such time-dependent tools were not originally developed for modelling fluctuations of the neutron flux, but a few recent attempts were reported in the literature [3][4]. There is thus a need to demonstrate whether such time-dependent tools provide physically-sound results, using as a reference the frequency-based tools.

Despite those recent attempts, there has not been any validation of existing time-domain tools for neutron noise calculations. This paper represents a first attempt to make such a validation, by comparing time-domain simulations using existing time-domain codes to frequency-domain simulations using dedicated neutron noise-based codes.

The tools chosen in this work are the time-domain-based code PARCSv3.2 [5], which is the NRC reference neutron diffusion code, and the frequency-domain tool CORE SIM [6], a neutron noise simulator developed by Chalmers University of Technology. CORE SIM was specifically developed for neutron noise calculations and was successfully benchmarked [7] against analytical or semianalytical reference solutions. Two different types of perturbations are considered for such comparisons in this paper: a point-like source, corresponding to the fluctuations created by an absorber of variable strength, and a traveling perturbation, simulating a perturbation in the coolant flow travelling upwards along a fuel channel. The comparisons require the development of an intricate methodology for the time-domain simulation data, involving source code modifications and the creation of new external supporting tools.

As will be illustrated in this paper, CORE SIM relies on first-order theory, i.e. the effect of nonlinearities are not taken into account. On the other hand, PARCS does not rely on any such approximation. In case of small perturbations, non-linear effects are negligible. When large perturbations/oscillations are encountered, as might be the case in Boiling Water Reactor during instability events, a non-linear code would need to be used. Large amplitude neutron noise was also observed in KWU-type PWRs [8], for which the use of a non-linear framework might be more suitable.

The paper is structured as follows. In section 2, the resolution of the neutron diffusion equation in the time- and frequency-domains is presented. In section 3, the methodology developed for the simulation of the neutron noise sources considered with each code is detailed. Finally, in the fourth section, the results are presented and discussed, and conclusions are drawn. 


\section{RESOLUTION OF THE NEUTRON DIFFUSION EQUATION}

\subsection{Time-dependent diffusion equation}

The diffusion equation with two energy groups and six groups of precursors of delayed neutrons is expressed, using standard notations, as follows [5]:

$$
\begin{gathered}
\frac{1}{v_{1}} \frac{\partial \phi_{1}(\boldsymbol{r}, t)}{\partial t}=-\nabla\left(-D_{1}(\boldsymbol{r}, t) \boldsymbol{\nabla} \phi_{1}(\boldsymbol{r}, t)\right)-\left(\Sigma_{a 1}(\boldsymbol{r}, t)+\Sigma_{12}(\boldsymbol{r}, t)\right) \phi_{1}(\boldsymbol{r}, t) \\
+(1-\beta) v \Sigma_{f 1}(\boldsymbol{r}, t) \phi_{1}(\boldsymbol{r}, t)+(1-\beta) v \Sigma_{f 2}(\boldsymbol{r}, t) \phi_{2}(\boldsymbol{r}, t)+\sum_{k=1}^{6} \lambda_{k} C_{k}(\boldsymbol{r}, t) \chi \\
\frac{1}{v_{2}} \frac{\partial \phi_{2}(\boldsymbol{r}, t)}{\partial t}=-\nabla\left(-D_{2}(\boldsymbol{r}, t) \nabla \phi_{2}(\boldsymbol{r}, t)\right)-\Sigma_{a 2}(\boldsymbol{r}, t) \phi_{2}(\boldsymbol{r}, t)+\Sigma_{12}(\boldsymbol{r}, t) \phi_{1}(\boldsymbol{r}, t) \\
\frac{\partial C_{k}(\boldsymbol{r}, t)}{\partial t}=\beta_{k} v \Sigma_{f 1} \phi_{1}(\boldsymbol{r}, t)+\beta_{k} v \Sigma_{f 2} \phi_{2}(\boldsymbol{r}, t)-\lambda_{k} C_{k}(\boldsymbol{r}, t) ; k=1, \ldots, 6
\end{gathered}
$$

Where the set of parameters and variables like diffusion coefficients, macroscopic cross-sections, thermal and fast fluxes and concentration of the six groups of neutron precursors, are space- and timedependent. Bold letters are used to indicate vectors.

The code PARCSv3.2 solves these equations for 3-dimensional systems, both in cartesian (as in the present study) or hexagonal geometries. Concerning the spatial discretization, different methods and options are available, such as, among others, the Finite Difference Method (FDM) or a nodal hybrid method combining the Analytical Nodal Method and the Nodal Expansion Method (HYBRID), in which case the Coarse Mesh Finite Difference scheme is used. The solution of the linear system [5] is obtained using a Krylov subspace.

\subsection{Frequency-domain diffusion equation}

In case of the frequency-domain and following the implementation used in CORE SIM, the balance equations are derived from two-group diffusion theory but considering a unique group of delayed neutrons [6]:

$$
\begin{gathered}
\frac{1}{v_{1}} \frac{\partial \phi_{1}(\boldsymbol{r}, t)}{\partial t}=-\nabla\left(-D_{1}(\mathbf{r}) \nabla \phi_{1}(\boldsymbol{r}, t)\right)-\left(\Sigma_{a 1}(\boldsymbol{r}, t)+\Sigma_{r}(\boldsymbol{r}, t)\right) \phi_{1}(\boldsymbol{r}, t) \\
+(1-\beta) v \Sigma_{f 1}(\boldsymbol{r}, t) \phi_{1}(\boldsymbol{r}, t)+(1-\beta) v \Sigma_{f 2}(\boldsymbol{r}, t) \phi_{2}(\boldsymbol{r}, t)+\lambda C(\boldsymbol{r}, t) \\
\frac{1}{v_{2}} \frac{\partial \phi_{2}(\boldsymbol{r}, t)}{\partial t}=-\nabla\left(-D_{2}(\boldsymbol{r}) \nabla \phi_{2}(\boldsymbol{r}, t)\right)-\Sigma_{a 2}(\boldsymbol{r}, t) \phi_{2}(\boldsymbol{r}, t)+\Sigma_{r}(\boldsymbol{r}, t) \phi_{1}(\boldsymbol{r}, t) \\
\frac{\partial C(\boldsymbol{r}, t)}{\partial t}=\beta v \Sigma_{f 1}(\boldsymbol{r}, t) \phi_{1}(\boldsymbol{r}, t)+\beta v \Sigma_{f 2}(\boldsymbol{r}, t) \phi_{2}(\boldsymbol{r}, t)-\lambda C(\boldsymbol{r}, t)
\end{gathered}
$$

It should be noted that in the equations above assume that the system is critical without the existence of any external neutron source and that the diffusion coefficients are time-independent. Neglecting the 
time-dependence of the diffusion coefficients was demonstrated to be acceptable for light water reactor systems [9].

The time-dependent terms are then expressed as the sum of the steady-state value (i.e. the mean value) and the fluctuations around the mean value (i.e. the so-called noise):

$$
X(\boldsymbol{r}, t)=X_{0}(\boldsymbol{r})+\delta X(\boldsymbol{r}, t)
$$

Applying a temporal Fourier transform, the following expression for the fluctuating part is obtained:

$$
\begin{gathered}
{\left[\nabla \cdot \overline{\bar{D}}(\boldsymbol{r}) \nabla+\overline{\bar{\Sigma}}_{d y n}^{c r i t}(\boldsymbol{r}, \omega)\right] \times\left[\begin{array}{l}
\delta \phi_{1}(\boldsymbol{r}, \omega) \\
\delta \phi_{2}(\boldsymbol{r}, \omega)
\end{array}\right]=} \\
\bar{\phi}_{r}(\boldsymbol{r}) \delta \Sigma_{r}(\boldsymbol{r}, \omega)+\overline{\bar{\phi}}_{a}(\boldsymbol{r})\left[\begin{array}{l}
\delta \Sigma_{a 1}(\boldsymbol{r}, \omega) \\
\delta \Sigma_{a 2}(\boldsymbol{r}, \omega)
\end{array}\right]+\overline{\bar{\phi}}_{f}^{c r i t}(\boldsymbol{r}, \omega)\left[\begin{array}{l}
\delta v \Sigma_{f 1}(\boldsymbol{r}, \omega) \\
\delta v \Sigma_{f 2}(\boldsymbol{r}, \omega)
\end{array}\right]
\end{gathered}
$$

where the meaning of the different terms can be found elsewhere, for instance in [6]. The neutron noise simulator tool CORE SIM solves these 3D equations in Matlab using a LU PQ decomposition. It should also be mentioned that prior to performing noise calculations, CORE SIM obtains the static neutron flux and the corresponding eigenvalue using either the explicitly-restarted Arnoldi method or the power iteration method with Wielandt's shift technique [10]. The computed eigenvalue is used to rescale the prompt neutron contributions, thus the terms $v \Sigma_{f}$ are divided by the eigenvalue to ensure no drift in the mean.

\section{METHODOLOGY}

In the present work, the methodology developed for comparing the results of the neutronics tools PARCSv3.2 and CORE SIM and originally developed by the present authors in [11] is detailed.

The case that will serve as the basis for this study corresponds to the neutronic configuration of a KWU nuclear reactor core.

\subsection{Methodology for steady-state cases}

Before performing noise calculations, it has to be verified that the two codes used provide consistent results for the nominal steady-state core configuration.

The PARCS model and input file were first defined. An equivalent CORE SIM model was then derived. The input data needed by CORE SIM are the dimensions of an elementary node in the $x$-, $y$-, and $z$ directions (in the present case, the size of every node is 23,23 and $10.625 \mathrm{~cm}$ along each respective direction) and the value of the different cross-sections for each node. Based on these data, CORE SIM solves the diffusion equation for the modelled reactor containing as many nodes as non-zero entries in the arrays of the cross-section sets.

The set of cross-sections were acquired from PARCS runs by editing the cross-sections retrieved from the cross-section set files (nemtab/r files) at the corresponding thermal-hydraulic steady-state conditions. These nemtab/r files were obtained using the SIMTAB methodology [12], which allows generating the files from the output of SIMULATE-3 [13].

For that purpose of obtaining the cross-sections for CORE SIM as well, the values of the diffusion coefficients and absorption, nu-fission and removal cross-sections, for two energy groups, read by PARCS were printed to an external file making use of some modifications implemented in the source 
code. Once printed, the data were reordered and written in a format directly readable in Matlab and CORE SIM. A program was created for this specific task.

Moreover, some preliminary tests were carried out to verify whether the cross-sections printed by PARCS included the correction due to the presence of Xenon in the reactor. This verification was carried out by comparing the CORE SIM steady-state results using sets of cross section data with or without activating the Xenon option in PARCS.

\subsection{Methodology for dynamical cases}

As regards the implementation of dynamical cases, two types of problems were investigated:

- Case of an absorber of variable strength.

- Case of a travelling perturbation in the coolant density from the inlet to the outlet of a given fuel assembly.

The main difference between both cases is that in the first case the disturbance is restricted to a single node where the fast and thermal absorption cross-sections are disturbed according to a sine function whose frequency, amplitude, phase and location of the applied perturbation in each case are defined by the user, and for which the amplitude and phase of the disturbance can be arbitrarily set. On the other hand, in the case of a disturbance caused by a travelling perturbation in the density, the determination of the amplitude and phase of the applied perturbation is slightly more complicated, as explained hereafter.

It must be emphasized that CORE SIM is only capable of defining a perturbation in terms of crosssections fluctuations. While the CORE SIM tool is explicitly designed to perform the simulation of neutron noise, this is not the case for PARCS. Modelling this type of dynamical systems is thus an issue which requires the development of an entire methodology involving changes in the source code and the development of certain functions and tools to automate the required tasks .

Since CORE SIM only uses one group of precursors of delayed neutrons, the same number of groups was adopted in PARCS taking the corresponding values for the fraction of delayed neutrons and the decay constant of the precursors as provided by SIMULATE-3.

\subsubsection{Absorber of variable strength}

The problem of an absorber of variable strength is by far the simplest problem of the two considered in this work. Due to the definition of the problem itself, the disturbance is restricted to a unique node for which only the values corresponding to the absorption cross section are modified in both energy groups.

The disturbance is induced as a function of time in the perturbed (fast and thermal) cross-sections as:

$$
\Sigma=\Sigma_{0}(1+A * \sin (\omega t))
$$

where $\Sigma$ is the perturbed cross section, $\Sigma_{0}$ is the unperturbed (mean) value, $A$ the amplitude and $\omega$ the desired angular frequency. To implement such a disturbance, the PARCS source code was modified, so that the fast and thermal absorption cross-sections for a certain cell could be directly defined by the user in each case. It should be mentioned that what matters for the calculation of the induced neutron noise is the resulting contributions of the individual types of cross-section perturbations to the applied noise sources in the fast and thermal energy groups, not so much the actual types of cross-sections being perturbed, as the right-hand side of Eq. (8) demonstrates. This explains why, for the absorber of variable strength, considering only a perturbation in the fast and thermal absorption cross-section is deemed sufficient to catch the physics at hand.

The generation of an input file to CORE SIM for the absorber of variable strength case is relatively simple. In addition to the data necessary to define the stationary case, only the following is needed: 
effective fraction of delayed neutrons and the corresponding decay constant, and the frequency of the perturbation. The noise source is then simply defined by an arbitrary amplitude for the perturbed crosssections in the corresponding node. Due to the heterogeneous nature of the system of equations being solved, the induced neutron noise is directly proportional to the applied perturbation, explaining why the amplitude can be arbitrary chosen (and the phase set to zero to simplify even further the case).

\subsubsection{Travelling perturbation}

In this case, the perturbation is introduced by the oscillation of the coolant density in an entire channel according to the following manner:

$$
\operatorname{DENS}_{i, k}=\operatorname{DENS}_{0_{i, k}}(1+A * \sin (\omega t+\varphi))=\operatorname{DENS}_{0_{i, k}}(1+A * \sin (\omega(t-\mathrm{k} * \Delta x / v)))
$$

$\operatorname{DENS}_{i, k}$ and $\mathrm{DENS}_{0_{i, k}}$ represent the values of the perturbed and unperturbed values of the density on each radial location $i$ and axial level $\mathrm{k}$, respectively. Due to the travelling nature of the perturbation, an axial phase shift $\varphi$ between nodes exists. This axial phase shift can also be expressed as a function of the node axial position $(\mathrm{k})$, the node height $(\Delta x)$ and by the speed at which the fluid is travelling upwards in the channel $(v)$.

However, including this perturbation is not a simple task, since it requires making changes to the source code of PARCSv3.2 and, subsequently, to the printing of the cross-sections interpreted by the code. This second step is essential because CORE SIM is only able to process nodal cross-sections as input. As earlier stated, CORE SIM does not consider fluctuations of the diffusion coefficients, which is a reliable enough approximation [9] for the frequency-domain calculations.

Also, obtaining the amplitude of the oscillation for each cross-section is difficult because the density affects all cross-sections and their response is different depending on the node. For this reason, a tool was developed. Knowing the actual oscillations at each point (which is an input data corresponding to the applied disturbance in density), the tool allows determining the resulting amplitude of the fluctuations in each cross-section for every time step. These local amplitudes are subsequently timeaveraged in order to overcome deviations due to the originally tabulated data or spurious values. Finally, the averaged amplitudes of each cross-section are introduced as input data in CORE SIM.

Finally, once the amplitude of each cross-section has been obtained for each node in the disturbed channel, it is introduced into the CORE SIM input file. Due to the axial time delay of the applied perturbation, the corresponding phase in the frequency domain is correspondingly expressed as an exponential function, thus leading to:

$$
G_{i, \text { frec }}=G_{i, \text { temp }} * e^{-i * \omega * \Delta x * \frac{k}{v}}
$$

where $G_{i, \text { frec }}$ is the complex number associated to the amplitude and the phase of each cross-section perturbation in the frequency domain and $G_{i, t e m p}$ is the amplitude in the temporal domain.

\section{RESULTS AND DISCUSSION}

In this section, a comparison between the results obtained by PARCSv3.2 and CORE SIM are showed and commented. Steady-state simulations are first analyzed, followed by the results for the dynamical cases corresponding to the cases studied: a variable strength absorber and a travelling perturbation. 


\subsection{Steady-state results}

The purpose of the initial steady-state comparison between PARCSv3.2 and CORE SIM is to clarify the appropriate numerical method to be used in PARCSv3.2 so that a faithful comparison for the simulations of perturbations can be carried out. The results are compared with the reference values provided by SIMULATE-3 since this model was provided by the nuclear power plant and was used to provide sets of cross-sections to both PARCS and CORE SIM. In addition, the level of necessary mesh refinement in CORE SIM is assessed.

Comparisons of $k_{\text {eff }}$ and the axial flux profiles are reported hereafter. Several options were tested: the two types of spatial discretization schemes in PARCSv3.2 (FDM or HYBRID), as well as two meshes in CORE SIM (a mesh identical to the one used in PARCSv3.2 and a refined mesh obtained by splitting each mesh cell in a given direction in two mesh cells). Assembly Discontinuity Factors (ADFs) were not considered in PARCSv3.2 because CORE SIM cannot account for ADFs at present.

Table I. Results of the comparison for k-effective.

\begin{tabular}{|c|c|c|c|c|c|}
\cline { 2 - 6 } \multicolumn{1}{c|}{} & $\begin{array}{c}\text { SIMULATE- } \\
\mathbf{3}\end{array}$ & $\begin{array}{c}\text { PARCS } \\
\text { Hybrid }\end{array}$ & PARCS FDM & CORE SIM & $\begin{array}{c}\text { CORE SIM } \\
\text { (double) }\end{array}$ \\
\hline k-effective: & 1.00077 & 1.00045 & 1.02221 & 1.02234 & 1.00525 \\
\hline \multicolumn{2}{|c|}{ pcm (vs SIMULATE-3) } & 31.9 & -2144.1 & -2156.6 & -447.677 \\
\hline
\end{tabular}

As we can see in Table I, the absolute differences in $k_{\text {eff }}$ (expressed in $p c m$ ) obtained for PARCSv3.2 and CORE SIM versus the reference value, are reasonably small when the Hybrid numeric scheme and the refined mesh are used in PARCSv3.2 and CORE SIM, respectively.

It can be observed in Figures 1 and 2, where the axial fluxes are normalized to the number of axial nodes, that a better match with the reference profile is obtained when Hybrid is chosen as PARCS nodal kernel and when a double mesh for CORE SIM is used. It should also be noticed that the results using the simplest mesh in CORE SIM and FDM method in PARCSv3.2 seems to be relatively close, both having a more bottom-peaked distribution as compared to the other cases. This is explained by the fact that CORE SIM is also based on finite differences. This discretization method requires a small mesh size in order to give accurate results.

Based on these results, in the following of this study, the options giving a better agreement with the reference results are chosen: the Hybrid option for PARCSv3.2 and the refined mesh in CORE SIM.

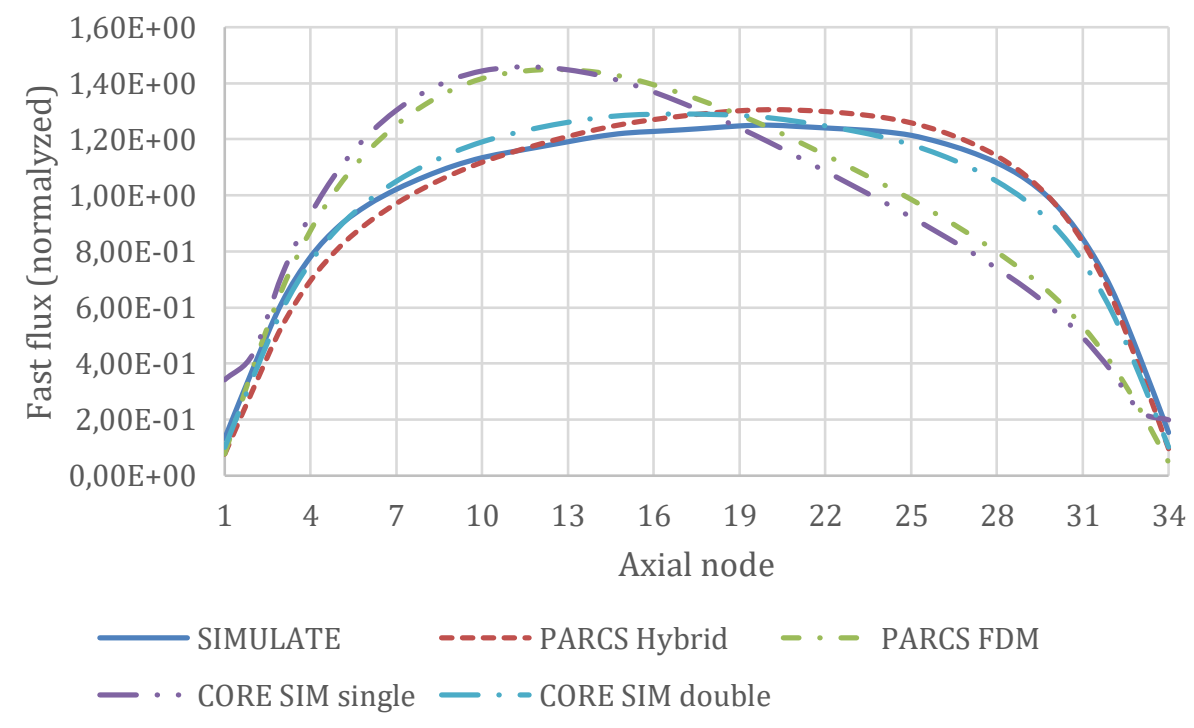


Figure 1. Comparison of the fast flux profiles using SIMULATE-3, PARCSv3.2 and CORE SIM.

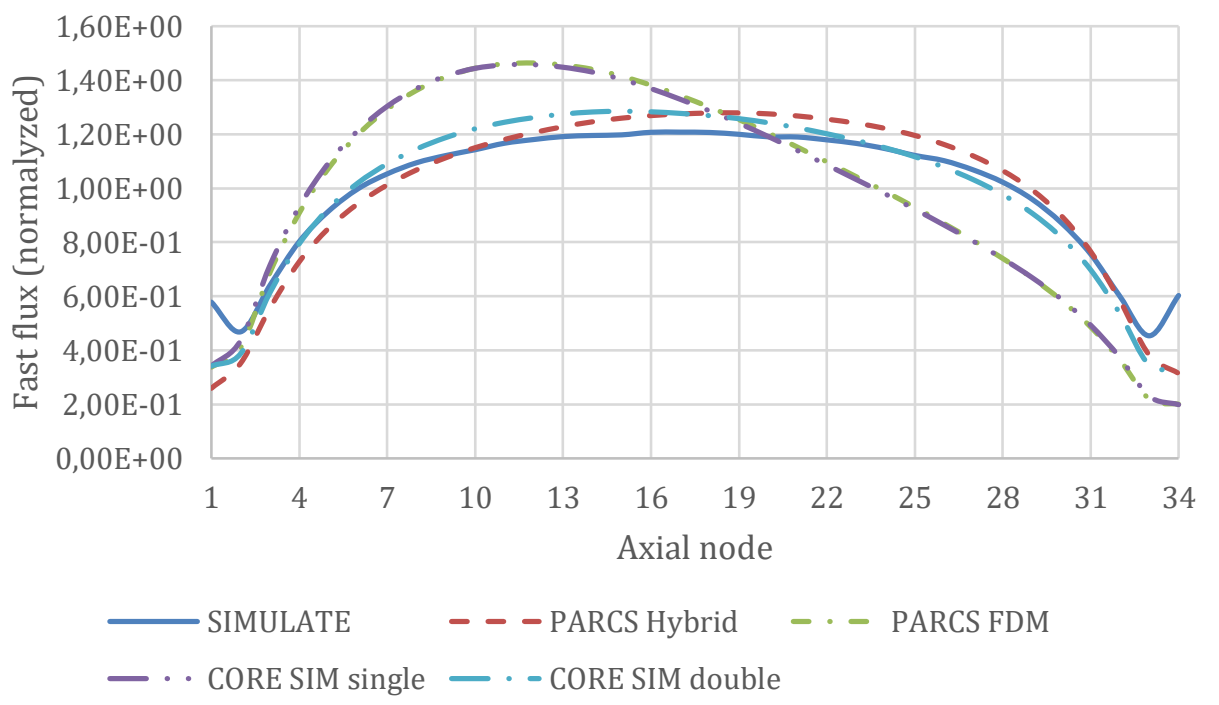

Figure 2. Comparison of the thermal flux profiles using SIMULATE-3, PARCSv3.2 and CORE SIM.

\subsection{Dynamical results}

Once the steady-state results are obtained and the models in CORE SIM and PARCSv3.2 chosen, the dynamical simulations were undertaken. As earlier mentioned, two cases are considered: an absorber of variable strength and a travelling perturbation. For each case, a few scenarios are considered in order to test the influence of:

- The frequency of the perturbation: $0.1 \mathrm{~Hz}, 0.5 \mathrm{~Hz}, 1 \mathrm{~Hz}, 2 \mathrm{~Hz}, 4 \mathrm{~Hz}, 5 \mathrm{~Hz}$ or $10 \mathrm{~Hz}$,

- The amplitude of the perturbation: $0.1 \%, 1 \%$ or $5 \%$,

- And the location of the perturbation: three different positions, from close to the center to the periphery of the core and located in the upper-left quarter of the core, as indicated in Fig. 3.

The aim of making these comparisons is to investigate the possible dependence of the accuracy of the simulations onto frequency, amplitude and location of the perturbation. Moreover, the neutron noise response is known to vary significantly with frequency [14] and location of the noise source, , therefore further justifying the considered cases. The location of the noise source refers to the radial position of the source: in the case of the travelling perturbation, all the axial nodes in a selected fuel channel are affected, meanwhile in the case of an absorber of variable strength, the source is confined only to the $10^{\text {th }}$ axial node from the bottom of the core (of 34 possible levels). The dimensions of the entire model in PARCS are $17 \times 17 \times 34$ meshes in the $x$-, $y$-, and $z$-directions, respectively.

The first objective of this study is to check whether similar trends are obtained in both codes. The results are thus presented throughout the following pages as spatial variations, in terms of amplitude and phase, of the results along the radial directions that embed all three possible positions of the perturbation, i.e. the eighth row in the core, and at different axial elevations. Moreover, a third graphic showing the axial variation of the amplitude is presented for the cases in which a travelling perturbation is studied. In the following, representative cases of each neutron noise source calculations are reported. Moreover, a case showing the limitations of the methodology is also shown. Finally, a compilation of all numerical results is presented.

Since different frequencies are considered, distinct time step and transient duration are chosen to accurately reproduce the desired sinusoidal perturbation in every case. Thus, for each frequency, the 
time step is selected so that every period is represented by 100 points. That is, we choose the hundredth part of the time period as time step. Moreover, 12 periods were considered so that enough periods allow overcoming possible problems when we apply the Fast Fourier Transform in the time-to-frequencydomain transformation, as explained later.

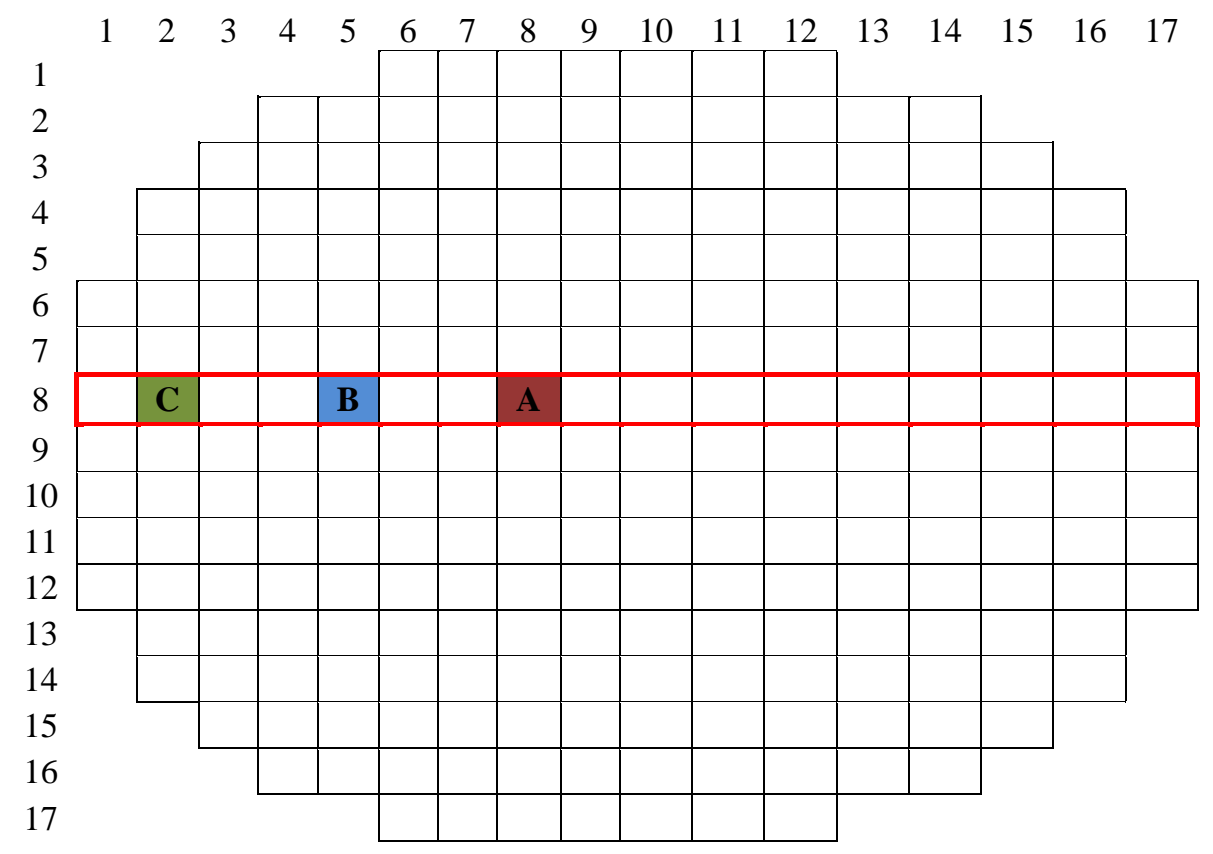

Figure 3. Scheme of the core showing location of the three positions studied.

The different nature of the tools used in the comparison requires treating the respective code outputs differently. Thus, meanwhile the results in PARCS are absolute values of the flux, the CORE SIM outcome is presented as separate variables, stationary (i.e. static) neutron flux and neutron noise, expressed as complex numbers. It is therefore necessary to subtract from the time-dependent PARCS fluxes the stationary value in order to compare only the variation provoked by the neutron noise sources described above. The presentation of the results in the frequency-domain in terms of amplitude and phase being most useful, the time-domain results are converted to the frequency-domain.

However, this conversion and the associated tasks to condition both sets of results for the purpose of comparisons requires specific tools that were developed in Matlab. A Fast Fourier Transform is applied to the time-domain results in order to obtain the amplitude and the phase of each cell. Besides, given the fact that each code has a different normalization, a rescaling of the results between the two codes is required. The rescaling is done by first obtaining, for each case, the relation for each node between the results of PARCS and CORE SIM. Subsequently, a global error minimization renormalization procedure is applied, by renormalizing the mean value of these relations and applying this renormalization to all nodes. This process is the reason why, as will be noticed in the following figures, the errors are largest in the vicinity of the applied perturbation.

It should be noticed that in the following figures the values presented for the amplitude correspond to the normalized and converted outcome of the codes following the methodology explained above. On the other hand, the phase values are defined in relation to the applied sinusoidal perturbation. Thereby, the phase differences correspond to the absolute differences between PARCS and CORE SIM, while the amplitude differences showed are relative differences obtained following equation 12. However, since the values of the flux are in some cases extremely small, they lead to relatively large differences, even for not so large absolute errors. An algorithm has thus been implemented in order to detect if some unreasonably large relative errors are obtained. If this is indeed the case, the order between PARCS and CORE SIM was changed in equation 12 for all the nodes. That is, in certain cases, the relative error 
is obtained using either CORE SIM or PARCS as the reference. Nevertheless, once the choice of the reference solution was made, the same rule was applied to all the nodes in the corresponding case.

$$
\text { Relative differences }=\frac{[\text { PARCS }]-[\text { CORE SIM }]}{[\text { CORE SIM }]}
$$

The first representative figures correspond to the absorber of variable strength and give the thermal flux for the case corresponding to the position $\mathrm{C}$, at $5 \mathrm{~Hz}$ and $5 \%$ of amplitude. This perturbation is confined to the $10^{\text {th }}$ axial level and to a given radial location. Figures 4 and 5 depict the spatial variation of the amplitude and the phase, respectively, along the chosen direction (highlighted in red in Figure 3).

As can be seen, a good fit is demonstrated for the spatial distribution of these parameters, thus leading to a low error. Only appreciable differences can be noticed at the position of the applied noise source. The results corresponding to the fast flux (not reported here) are equivalent.

Similar assessments are obtained for all the cases related to the absorber of variable strength. As can be seen in Figures 12 and 13, which summarize for every case considered in the present work the maximum and RMS errors for the amplitude and the phase, respectively, the relative errors obtained for all the cases are reliable (RMS around $10 \%$ ), which seems thus to be a common feature for the cases associated with the absorber of variable strength. Furthermore, there are no visual significant discrepancies between the time-domain and frequency-domain simulations when plotting the results together. However, the maximum relative errors in the amplitude are, for all cases, above $30 \%$. This is a rather big maximum relative error. Considering that the RMS error is small and the fact that the relative error tends to maximize the error when small values are compared, one concludes that such large deviations only occur in isolated locations.

It should be mentioned that some additional modifications had to be implemented in the PARCSv3.2 source code, in order to increase from 4 to 11 the number of significant digits used by PARCS to edit the flux values. This is motivated by the fact that PARCS models the total flux value, i.e. the sum between the mean value and the noise, without differentiating the two, as CORE SIM does. Without such a modification, the values extracted for the fluctuations would not be accurate enough and unphysical results in terms of induced neutron noise would be obtained. 

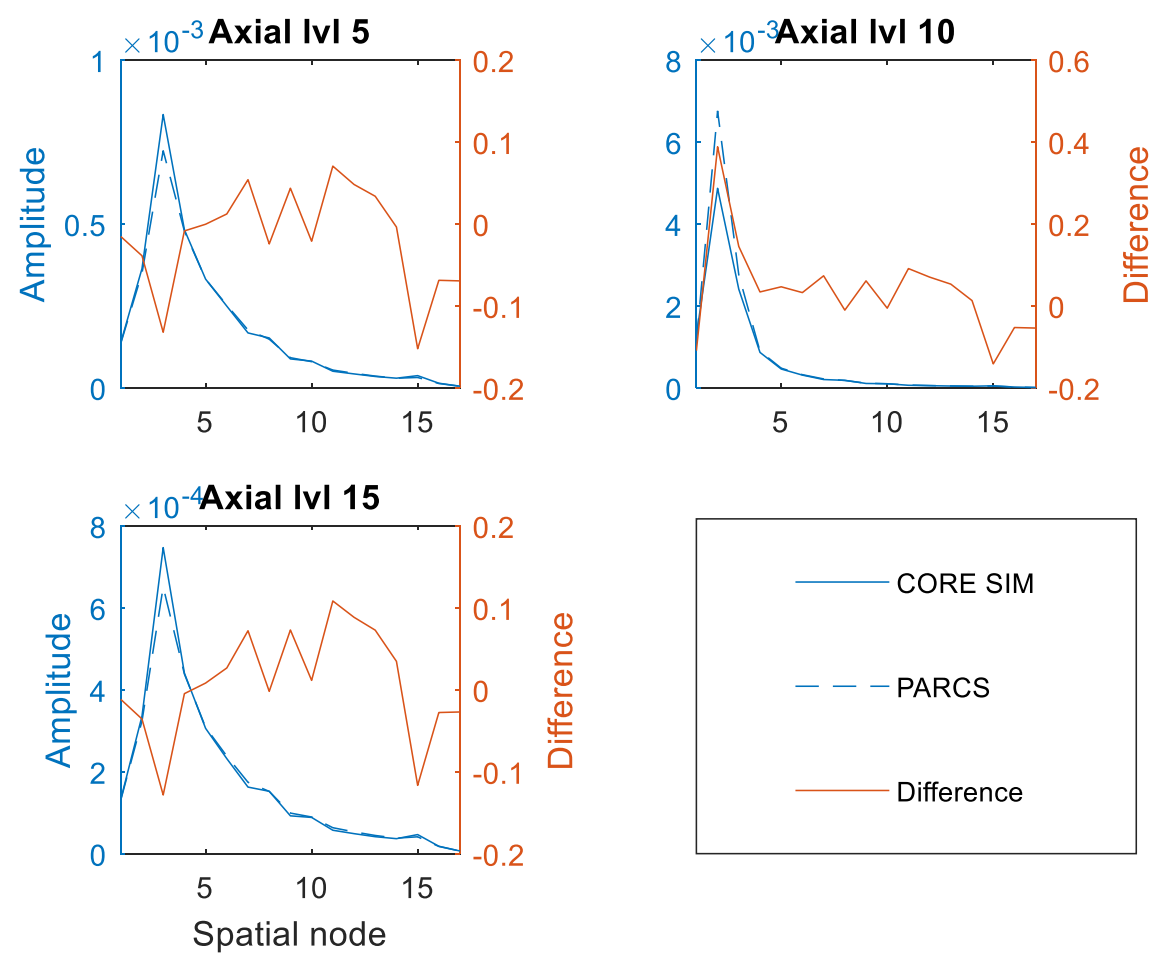

Figure 4. Amplitude of CORE SIM and PARCSv3.2 and corresponding relative error obtained between the tools for the case of an absorber of variable strength at $5 \mathrm{~Hz}$, located at point $\mathrm{C}$ with a $5 \%$ amplitude.
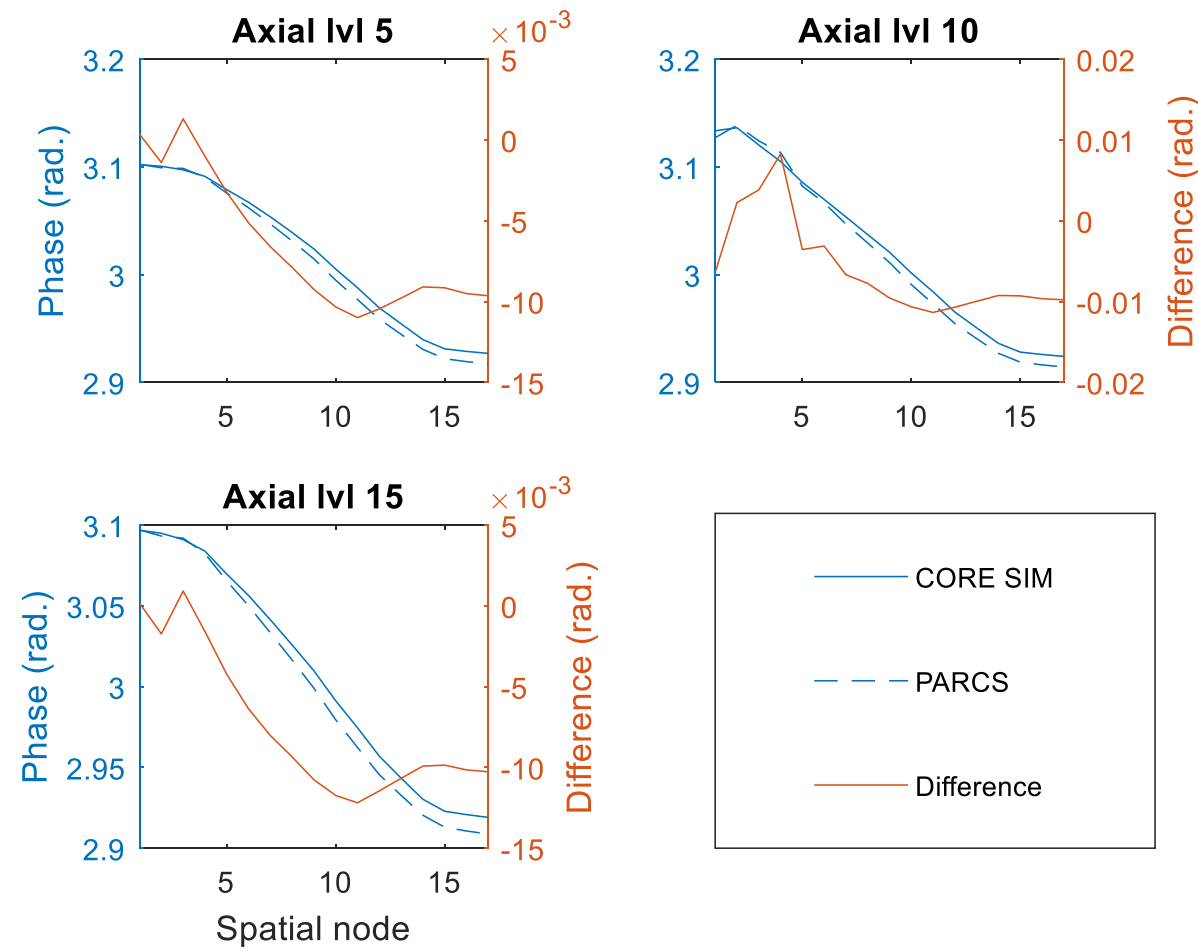

Figure 5. Phase of CORE SIM and PARCSv3.2 and corresponding absolute error obtained between the tools for the case of an absorber of variable strength at $5 \mathrm{~Hz}$, located at point $\mathrm{C}$ with a $5 \%$ amplitude. 

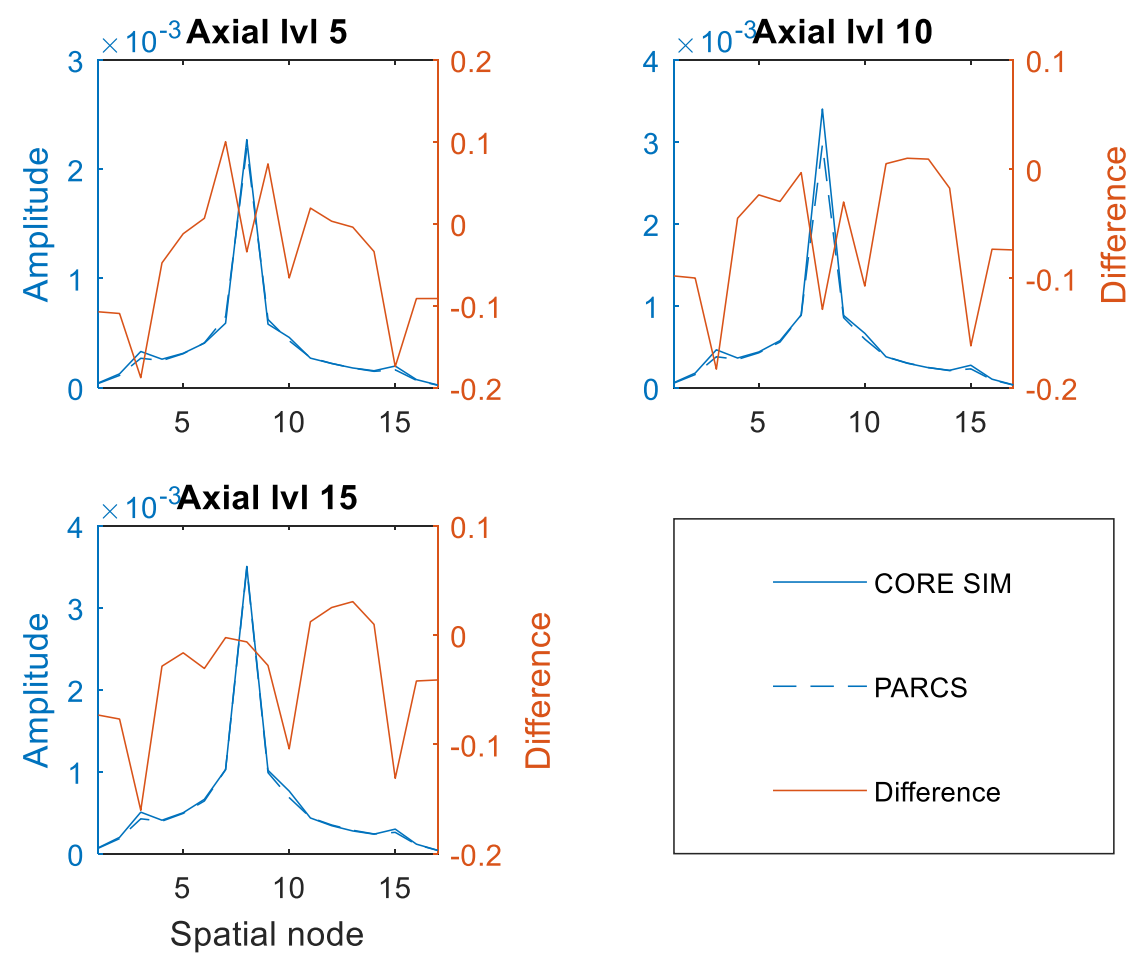

Figure 6. Amplitude of CORE SIM and PARCSv3.2 and corresponding relative error obtained between the tools for the case of a travelling perturbation at $0.5 \mathrm{~Hz}$, located at point $A$ with a $1 \%$ amplitude.
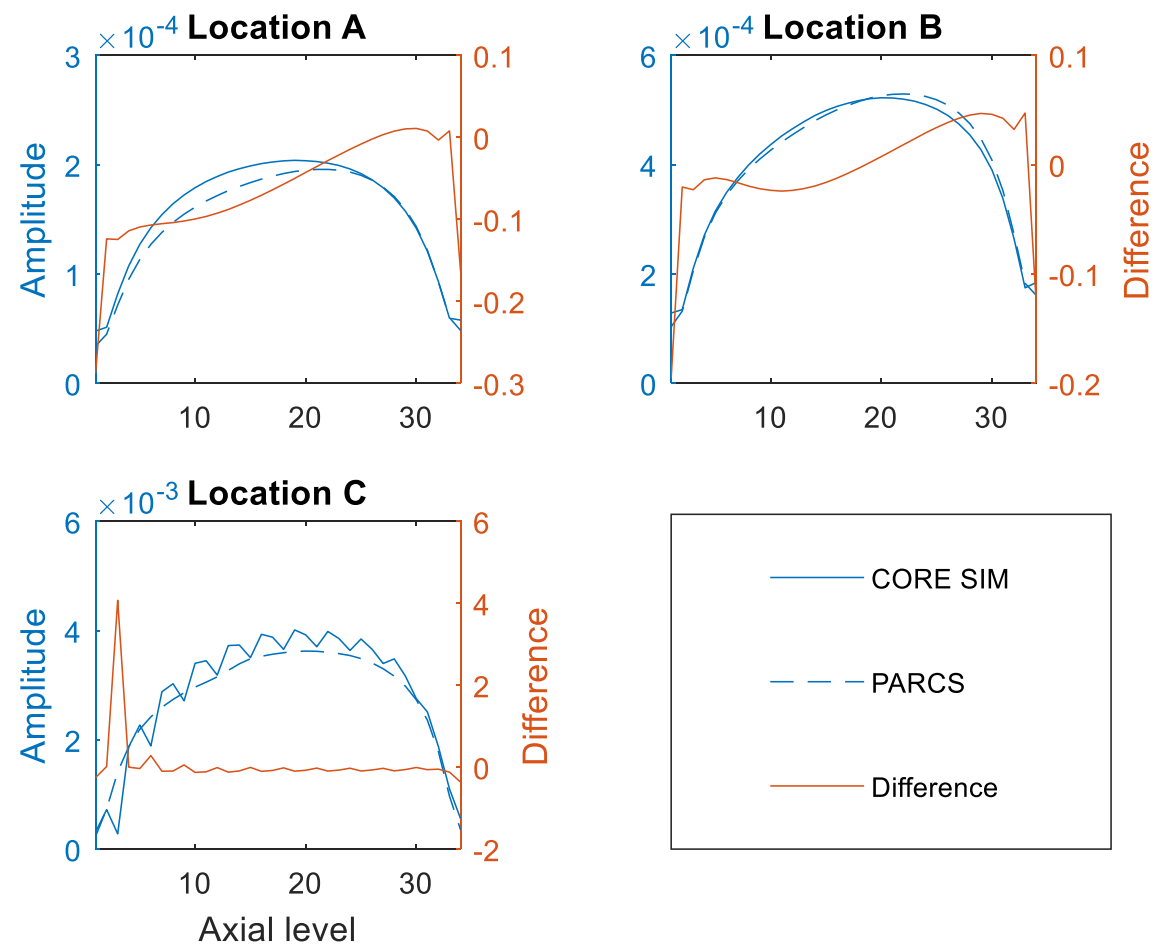

Figure 7. Axial variation of the amplitude of CORE SIM and PARCSv3.2 and corresponding relative error obtained between the tools for the case of a travelling perturbation at $0.5 \mathrm{~Hz}$, located at point $A$ with a $1 \%$ amplitude. 

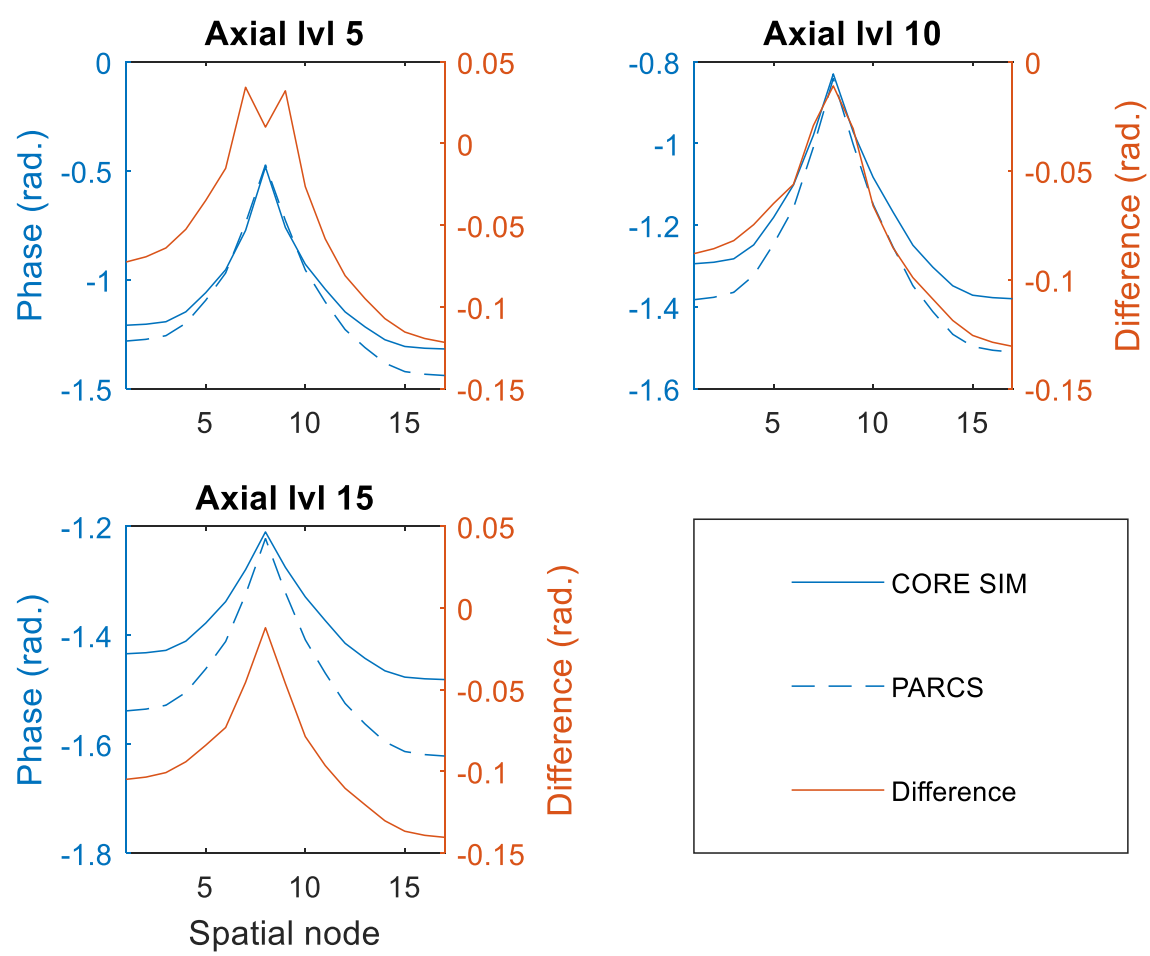

Figure 8. Phase of CORE SIM and PARCSv3.2 and corresponding absolute error obtained between the tools for the case of a travelling perturbation at $0.5 \mathrm{~Hz}$, located at point $A$ with a $1 \%$ amplitude.

In the case of the travelling perturbation, the agreement between both tools is bad for some cases and, in general, worse than the agreement obtained for the case of the absorber of variable strength. In order to better highlight the deterioration of the agreement between the time- and frequency-domain simulations, the dependency of the results with the location and frequency is explained in more details below. More precisely, two representative cases corresponding to a good agreement is presented, followed by a case leading to a worsening of the agreement between the two solutions.

First, the results of the spatial variation of the amplitude and phase along the mentioned line ( $8^{\text {th }}$ row in the radial configuration) and its axial variation for each position considered, for the case of a travelling perturbation on coolant density with a frequency of $0.5 \mathrm{~Hz}$, an amplitude of $1 \%$ and located at position A (i.e. close to the center of the core) are shown in figures 6 to 8, respectively. As can be seen, a good agreement between both solutions is observed for the amplitude, as well as for the phase of the neutron noise.

However, poorer agreement is obtained in other cases. Figures 9 to 11, corresponding to a travelling perturbation at $4 \mathrm{~Hz}$, with an amplitude of $1 \%$ and located at point $\mathrm{A}$, shows much higher discrepancies between the two codes, despite reproducing similar trends. Besides, the results of the time-domain simulations exhibit, in some cases, either a systematic overestimation or underestimation at a given axial level, as compared to the frequency-domain simulations.

Based on all the examined cases, a strong dependency with the frequency and location of the perturbation is observed for the travelling perturbation, as can be seen in Figures 10 and 11. On the other hand, not significant influence with the gain of the perturbation is perceived. 

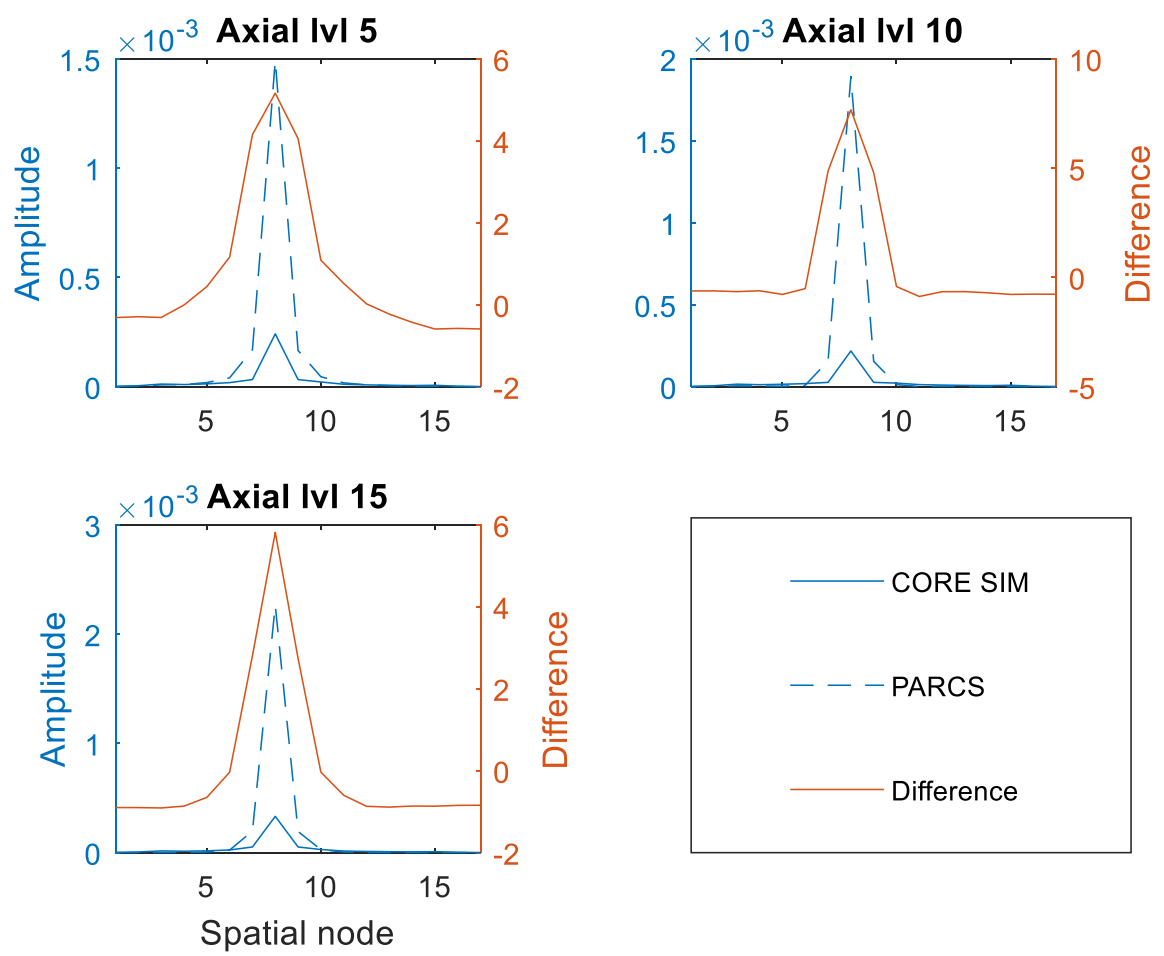

Figure 9. Amplitude of CORE SIM and PARCSv3.2 and corresponding relative error obtained between the tools for the case of a travelling perturbation at $4 \mathrm{~Hz}$, located at point $\mathrm{A}$ with a $1 \%$ amplitude.
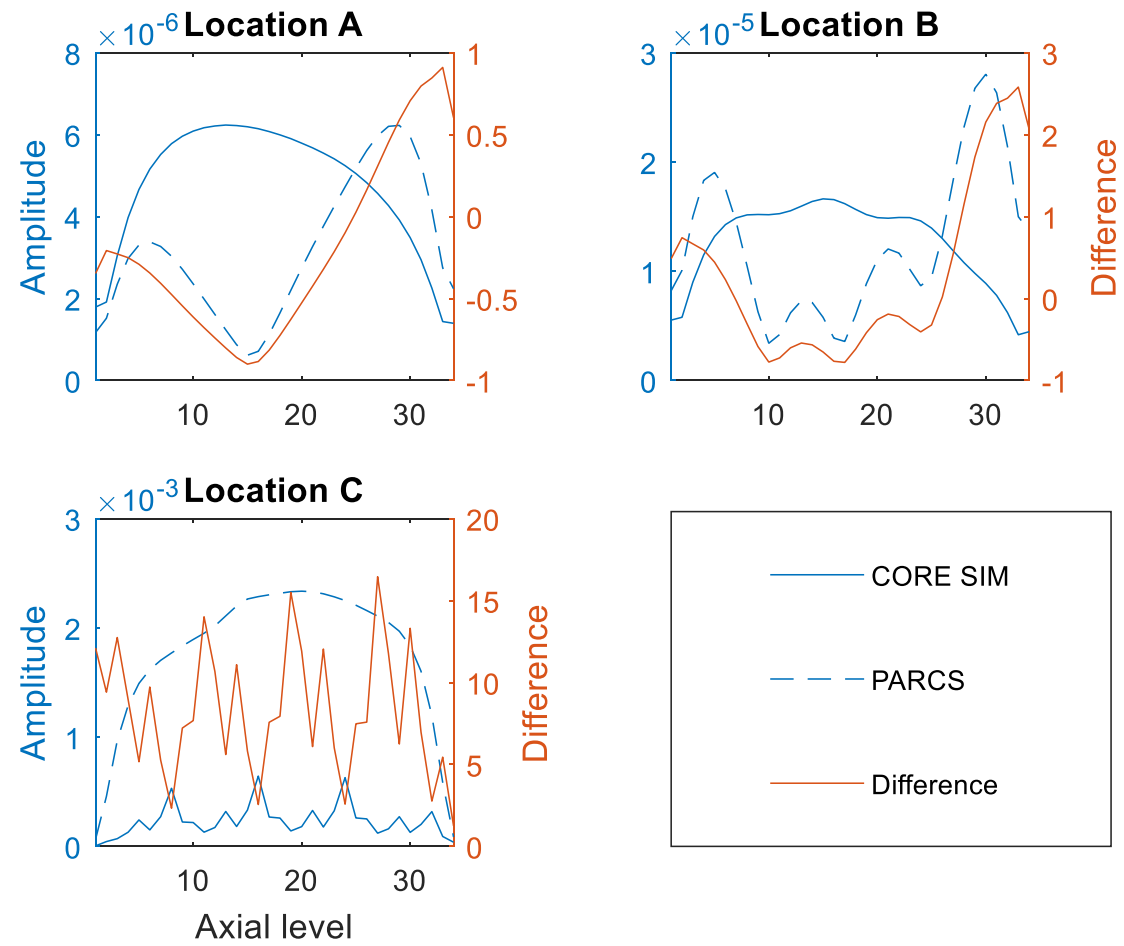

Figure 10. Axial variation of the amplitude of CORE SIM and PARCSv3.2 and corresponding relative error obtained between the tools for the case of a travelling perturbation at $4 \mathrm{~Hz}$, located at point A with a $1 \%$ amplitude. 

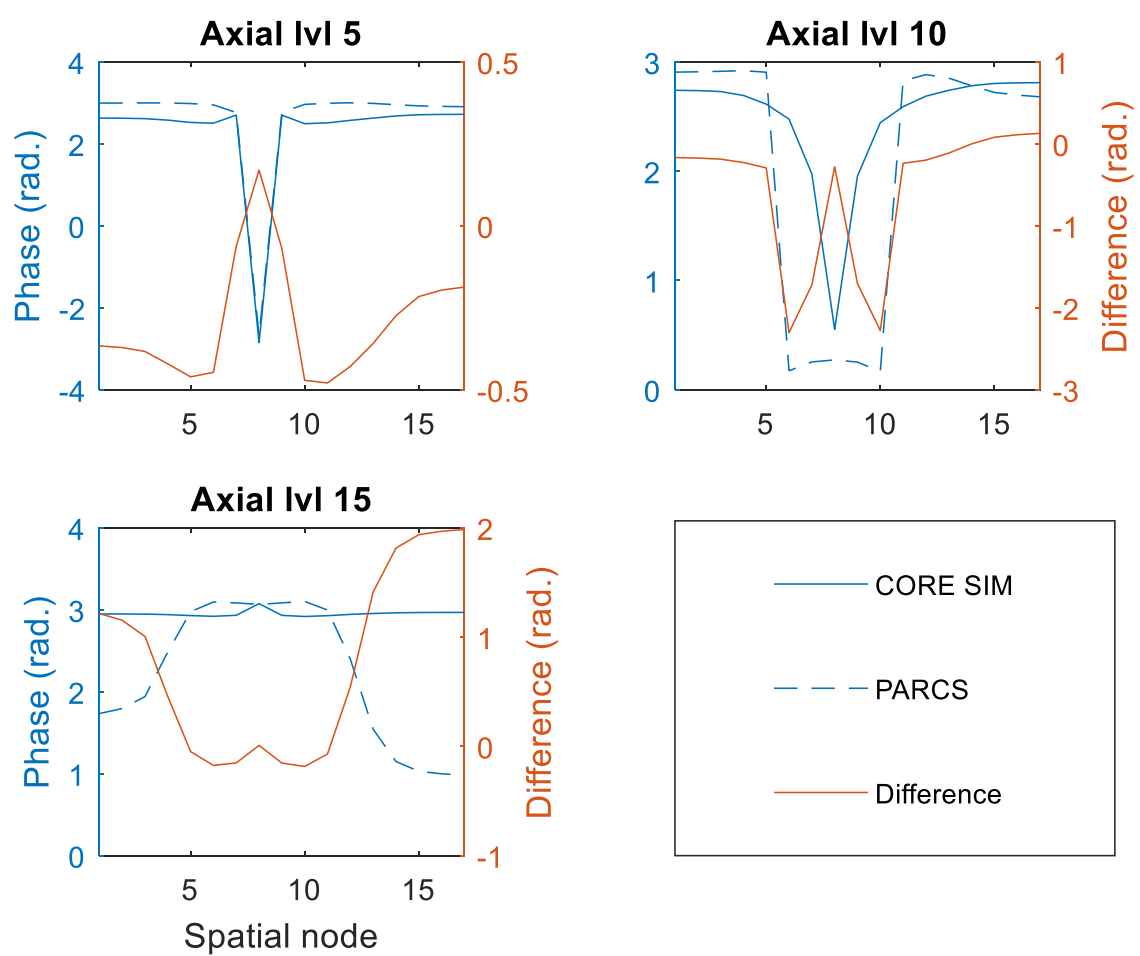

Figure 11. Phase of CORE SIM and PARCSv3.2 and corresponding absolute error obtained between the tools for the case of a travelling perturbation at $4 \mathrm{~Hz}$, located at point $\mathrm{A}$ with a $1 \%$ amplitude.

More specifically, confident results are obtained for the perturbation having a frequency equal to or lower than $1 \mathrm{~Hz}$, and located at position $\mathrm{A}$ or $\mathrm{C}$. For higher frequencies or location at position $\mathrm{B}$, some overestimation and underestimation of PARCS versus CORE SIM are observed, with a poor agreement between the two codes in most of the nodes.

This study represents a first evaluation of the capabilities of PARCSv3.2 to faithfully perform neutron noise calculations. It was noticed that the simulation of point-like perturbations, such as an absorber of variable strength, seem to provide much better agreement with the benchmarked frequency-domainbase CORE SIM tool, than for travelling perturbations along the core.

Finally, as earlier indicated, a compilation of figures that allows taking a quick grasp of the influence of the frequency, location and amplitude of the perturbation is provided in Figures 12 to 15. In these figures, we show the maximum errors and the RMS errors for all the simulations undertaken in this work. The errors corresponding to the amplitude are shown in relative terms (\%), which despite indicating very large deviations, is considered as a more appropriate way to compare the various simulations. It should be observed, as earlier stated, that most of the very large discrepancies are the consequence of very small values of the amplitude of the noise. The errors in the phase, on the other hand, are given in absolute terms, i.e. in radians.

The RMS error, found be around 10\% for the amplitude in most of the cases for the absorber of variable strength, stems from the inherent difficulty of comparing the results of time-domain simulations to the results of frequency-domain simulations. On the other hand, the error in the phase becomes lower and lower as the frequency increases, but remains appreciable. However, slightly larger errors are obtained for the cases in which the outermost location (position C) and the lowest amplitude are considered, even if the same trends are followed in all the cases. It is considered that the results qualitative fit to each other despite the differences between both calculation methods. It should be mentioned that in Figure 13 , of the four results listed in the legend, only two are visible. This is consequence of a perfect 
overlapping of the fast and thermal phase for each investigated case. A similar effect occurs in Figure 15 for the fast and thermal RMS of the phase.

Furthermore, good agreement is obtained for cases of the travelling perturbation if the frequencies are equal to or less than $1 \mathrm{~Hz}$ and, specially, if the perturbation is located close to the center of the core. For the other cases, the simulation of the travelling density perturbation could lead to large errors between the frequency-domain and time-domain codes, with the maximum and RMS differences taking large values. Thus, for the sake of clarity, the maximum errors were removed from the graphs. Moreover, large discrepancies for the phase are also observed.

Finally, it is somehow surprising that the agreement between PARCS and CORE SIM for the case of the travelling perturbation significantly deteriorates whereas the effect of a spatially-distributed noise source (as in the case of a travelling absorber) can be seen as a convolution integral between the neutron noise induced by point-like perturbations (as in the case of an absorber of variable strength) and the spatial distribution of the noise source [1]. 


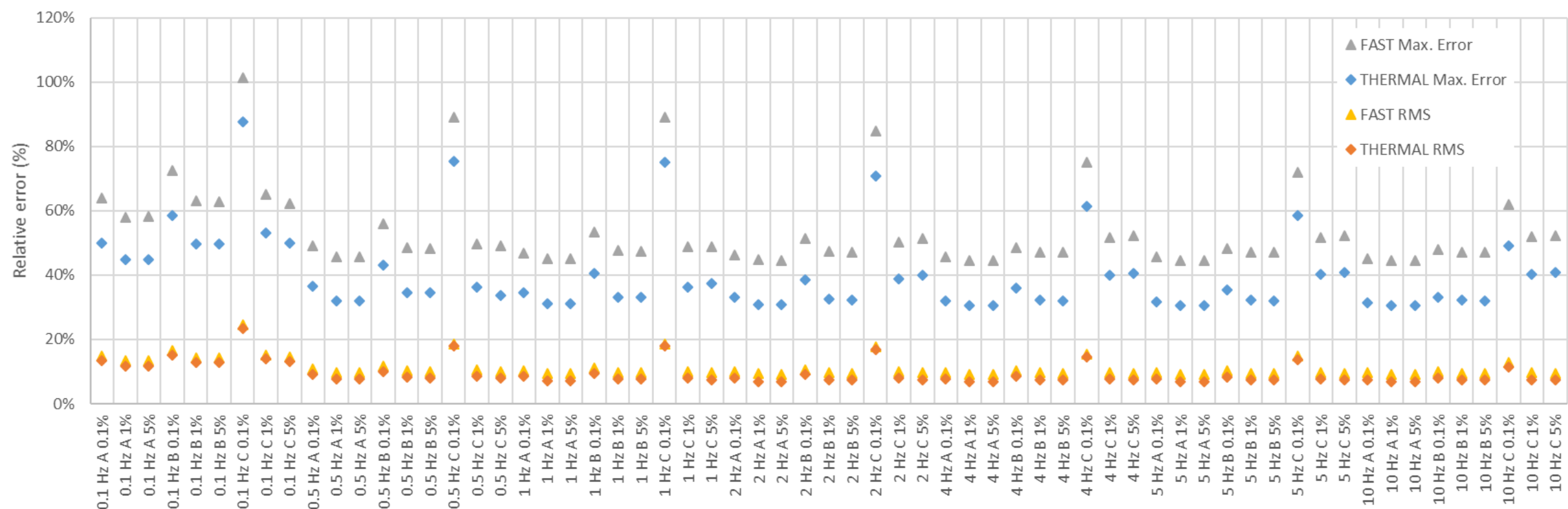

Figure 12. Relative errors, maximum and RMS, for the amplitude for the case of the absorber of variable strength.

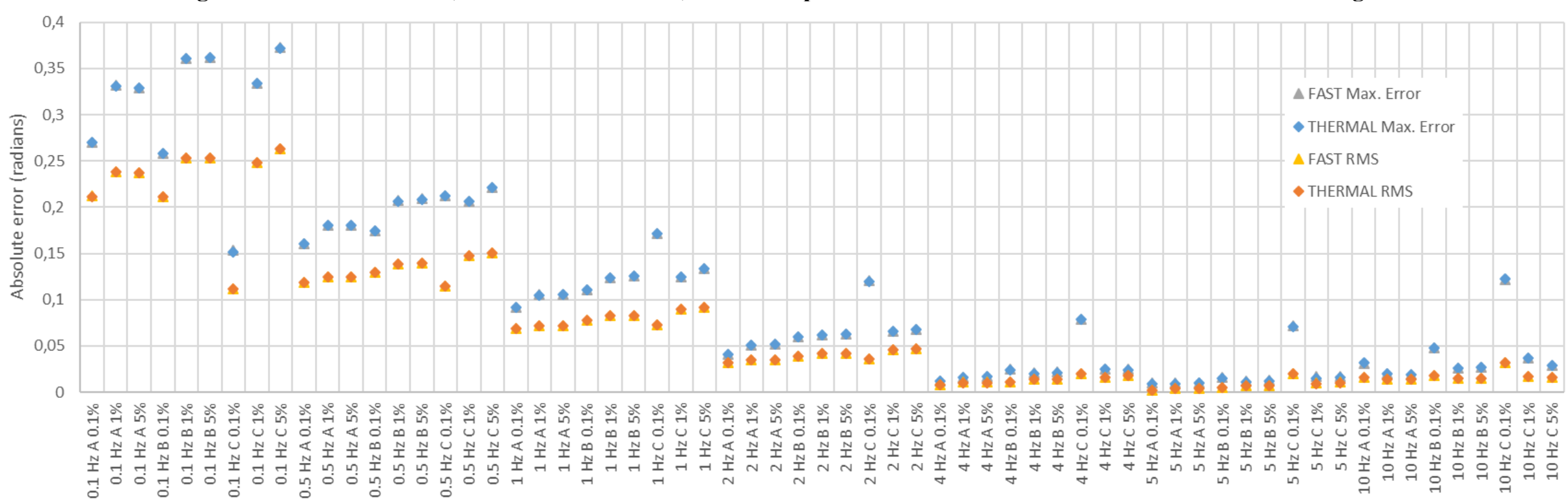

Figure 13. Absolute errors, maximum and RMS, for the phase for the case of the absorber of variable strength. 


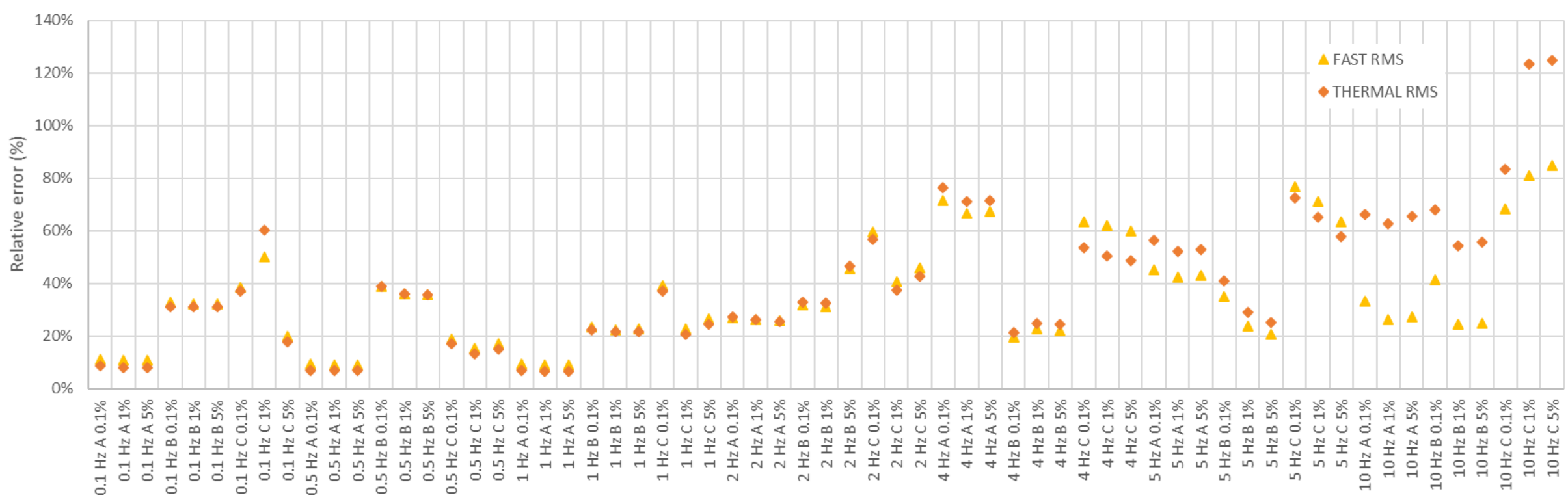

Figure 14. Relative RMS errors, for the variation of the amplitude for the case of the traveling perturbation.

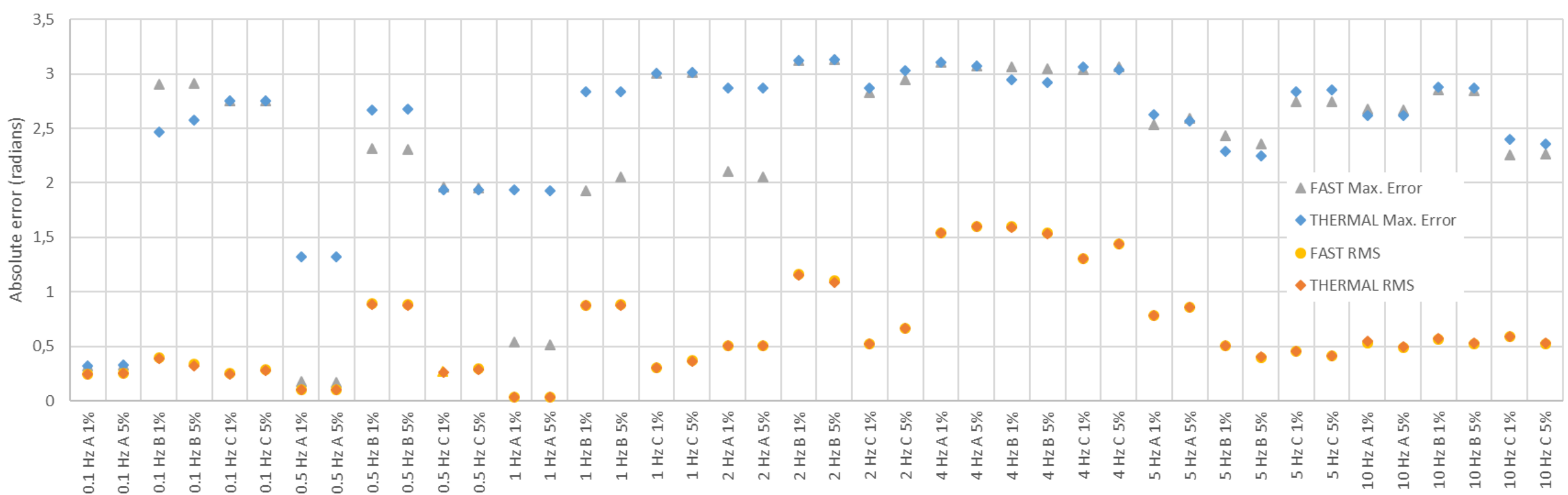

Figure 15. Absolute errors, maximum and RMS, for the variation of the amplitude for case of the traveling perturbation. 


\section{CONCLUSIONS}

In this work, a methodology for comparing the results of neutron noise simulations using the timedomain-based code PARCSv3.2 and the frequency-domain-based code CORE SIM is developed. This requires making changes to the PARCSv3.2 source code to: a) be able to introduce perturbations in the model, b) to edit the corresponding cross-sections and c) to edit the induced neutron flux with a significant number of digits.

The results obtained in this study demonstrate that PARCS, although not developed for that purpose, is able to provide a meaningful estimation of the induced neutron noise in some conditions. Thus, reliable results can be obtained in the frequency range $0.1-10 \mathrm{~Hz}$ in the case of a point-like source, such as an absorber of variable strength. For the case of a travelling perturbation, only reliable enough results are obtained if the frequency is equal to or lower than $1 \mathrm{~Hz}$ and if the location of the perturbation is close to the reactor center. In other cases, i.e. for higher frequencies or for outermost locations of the perturbation, an overestimation or underestimation of the PARCS results versus the CORE SIM results is demonstrated, leading to unacceptable results when using PARCS.

The developed methodology aimed at performing neutron noise calculations in PARCS must be further examined in order to identify the root cause of the large deviations observed, as for example, the influence of numerical diffusion induced by the numerical schemes available in PARCS.

\section{ACKNOWLEDGMENTS}

This work was carried out under the pre-doctoral contract FPI Subprogram 1 and mobility aids of the Universitat Politècnica de Valencia and the support of the Spanish Ministerio de Ciencia e Innovación under the project ENE2017-89029-P. The research leading to these results was also partially funded from the Euratom research and training programme 2014-2018 under grant agreement No 754316 (CORTEX project).

\section{REFERENCES}

1. I. Pázsit and C. Demazière, Noise techniques in nuclear systems, Chapter in the Handbook of Nuclear Engineering, D. Cacuci, Ed., ISBN 978-0-387-98150-5, Springer, Vol. 3 (2010).

2. Demazière C., Vinai P., Hursin M., Kollias S., and Herb J., "Overview of the CORTEX project", Proc. Int. Conf. Physics of Reactors - Reactor Physics paving the way towards more efficient systems (PHYSOR2018), Cancun, Mexico, April 22-26, 2018 (2018)

3. D. Chionis, A. Dokhane, L. Belblidia, M. Pecchia, G. Girardin, H. Ferroukhi, A. Pautz, "SIMULATE-3K Analyses of Neutron noise response to fuel assembly vibrations and thermalhydraulics parameters fluctuations", Int. Conf. Mathematics \& Computational Methods Applied to Nuclear Science \& Engineering (M\&C 2017), Jeju, Korea, April 16-20, 2017 (2017).

4. M. Viebach, N. Bernt, C. Lange, D. Hennig, A. Hurtado. "On the influence of dynamical fuel assembly deflections on the neutron noise level”. Prog. Nucl. Energy, 104 (2018), pp. 32-46.

5. T. Downar, Y. Xu, V. Seker. DRAFT (01/16/12) PARCSv3.0 U.S. NRC Core Neutronics Simulator THEORY MANUAL. Department of Nuclear Engineering and Radiological Sciences, University of Michigan, MI, USA (2012) 
6. C. Demazière, "CORE SIM: A multi-purpose neutronic tool for research and education," Annals of Nuclear Energy, 38 (12), pp. 2698-2718 (2011).

7. C. Demazière, Validation and demonstration of the CORE SIM neutronic tool. Report CTH-NT242, Chalmers University of Technology, Gothenburg, Sweden (2011).

8. Reactor Safety Commission (RSK), PWR neutron flux oscillations, RSK Statement (457th meeting on 11.04.2013). http://www.rskonline.de/en/meeting457.

9. V. Larsson and C. Demazière, "Comparative study of 2-group P1 and diffusion theories for the calculation of the neutron noise in 1D 2-region systems", Annals of Nuclear Energy, 36 (10), pp. 1574-1587 (2009).

10. C. Demazière, Description of the models and algorithms used in the CORE SIM neutronic tool. Report CTH-NT-241, Chalmers University of Technology, Gothenburg, Sweden (2011).

11. N. Olmo-Juan, C. Demazière, T. Barrachina, R. Miró, G. Verdú, "Comparative study of neutron noise calculations using the neutron kinetics code PARCS and the neutron noise simulator CORE SIM". Int. Conf. Reactor Physics paving the way towards more efficient systems (PHYSOR 2018), Cancun, Mexico, April 22-26, 2018

12. Roselló, O., 2004. Development of a methodology for generating cross sections to the simplification of the LWR core reactors and its application to neutronic-thermalhydraulic coupled codes [This document is only available in Spanish]. PhD Thesis, Universidad Politécnica de Valencia.

13. Covington, L.J., Cronin, J.T., Umbarger, J.A., "SIMULATE-3: advanced three-dimensional twogroup reactor analysis code”. Studsvik Scandpower, report Studsvik/SOA-95/15 Rev 2.

14. C. Demazière, V. Dykin and K. Jareteg, "Development of a point-kinetic verification scheme for nuclear reactor applications", Journal of Computational Physics, 339, pp. 396-411 (2017) 